\title{
Impacto del programa social Pensión 65 sobre el gasto en alimentos, 2017-2018
}

\author{
JUAN INQUILLA MAMANI \\ Universidad Nacional del Altiplano \\ inquilla@hotmail.com \\ ALFREDO PELAYO CALATAYUD MENDOZA \\ Universidad Nacional del Altiplano \\ alfredopelayo@yahoo.com
}

Resumen. El objetivo de este artículo es estimar el impacto del programa social Pensión 65 sobre el gasto per cápita en alimentos. El estudio se basa en una perspectiva metodológica cuantitativa, con la técnica de doble diferencia con propensity score matching (DD-PSM). Para este fin, se utilizan la base de datos de la Encuesta Nacional de Hogares (Enaho) 2017-2018, tanto para grupo de tratamiento como para el de control. La unidad de análisis son los hogares del Perú a nivel nacional que participan dos años consecutivos en el programa, y se toma como línea de base el año 2017. Los resultados obtenidos indican que el gasto per cápita en alimentos se ha incrementado en un 15,02\% para aquellos hogares beneficiarios del programa Pensión 65.

Palabras clave: Pensión 65; consumo per cápita; consumo de alimentos; método de doble diferencia; programas sociales; Perú.

\section{Impact of the Pensión 65 social program on spending on food, 2017-} 2018

Abstract. The objective of this article is to estimate the impact of the Pension 65 social program on per capita expenditures on food. The study is based on a quantitative methodological perspective, using the differences-in-differences technique with propensity score matching (DD-PSM). For this purpose, the database of the 2017-2018 National Household Survey (ENAHO) is used, both for the treatment and the control group. The unit of analysis are Peruvian households at the national level that participated in the program for two consecutive years, with 2017 as the baseline. The results obtained indicate that per capita expenditure on food has increased by $15.02 \%$ for those households that receive the Pensión 65 program. 
Apuntes 86, primer semestre 2020 / Inquilla y Calatayud

Keywords: Pensión 65; consumption per capita; food consumption; double difference method; social programmes; Peru. 


\section{Introducción}

El programa Pensión 65 tiene entre sus objetivos el de contribuir a mejorar el bienestar de la población de 65 a más años de edad que se encuentra en condición de pobreza extrema de acuerdo con la calificación socioeconómica del Sistema de Focalización de Hogares (Sisfoh). En general, las políticas públicas deben priorizar sus acciones para atender y focalizar claramente a la población objetivo en función de criterios de selección bien establecidos. En este contexto, el programa busca atender a la población adulta en situación de vulnerabilidad, y el beneficio consiste en la entrega de un subsidio de 250 soles por persona con una periodicidad de dos meses.

En este contexto, la Dirección de Calidad del Gasto Público del Ministerio de Economía y Finanzas (2015) realizó una evaluación de impacto del Programa Nacional de Asistencia Solidaria Pensión 65 y concluyó que, transcurridos tres ańos desde su implementación, el programa redujo la proporción de adultos mayores que realizan trabajos remunerados, lo que representa una disminución del $9 \%$ en el número de personas que trabajan por necesidad económica y, por tanto, una reducción del $22 \%$ en el ingreso laboral promedio. Por otra parte, se incrementó el consumo mensual per cápita del hogar en un 40\%; de dicho incremento, el $66 \%$ se destinó al consumo de alimentos y el 34\% a otros gastos, que conjuntamente permitieron aliviar la condición de vulnerabilidad en el corto plazo. En la misma perspectiva, otros estudios realizados muestran una reducción en la oferta de horas laborales y un aumento en las horas empleadas en ocio u otras actividades (Galiani, Gertler, \& Bando, 2014; Torres \& Salinas, 2016; Gondim Teixeira, 2009; Durán Valverde, Mendoza De Souza, \& Picado Chacón, 2009).

Por otra parte, las investigaciones empíricas a nivel internacional han encontrado evidencia de algún tipo de efecto negativo de una pensión no contributiva sobre la oferta laboral de los beneficiarios. En el caso de México, las evaluaciones del programa "70 y Más», por ejemplo, muestran una disminución del número de adultos mayores en trabajos remunerados (Galiani \& Gertler, 2012), e incluso se encontró que el programa había generado mayor bienestar social en adultos mayores que no presentaron disminución en su oferta laboral (Gertler et al., 2014). Por último, otros autores (Edmonds, 2006; Carvalho, 2012; Barrientos, 2003; Barrientos \& Lloyd-Sherlock, 2002) concluyen que las políticas de pensiones no contributivas no solo pueden incrementar el bienestar de los hogares a través de un aumento de las horas de ocio o de presencia en el hogar, sino que también pueden ser consideradas una forma de reducir la vulnerabilidad de los hogares. 
Asimismo, está el estudio realizado por Teixeira (2008), que muestra una reducción mínima en las horas de mano de obra provistas en respuesta a las transferencias realizadas por el programa. Dicho estudio sostiene que la reducción fue estadísticamente significativa, pero no fue grande. La variación es de entre 0,5 y 3,5 horas por semana para los adultos que trabajan. El efecto es mayor dependiendo de la proporción de la transferencia en los ingresos de los hogares. Aquellas personas que están por debajo del umbral de pobreza extrema reducen su oferta de mano de obra más que aquellas que se encuentran por debajo del umbral de pobreza. Además, los hogares en los que solo hay un niño o aquellos que reciben $R \$ 15,00,50,00$ o 65,00, reducen su oferta de mano de obra para el trabajo remunerado más que aquellos con familias más grandes.

Según Bosch y Guajardo (2012, citado por Torres y Salinas, 2016), se produjo una reducción en la participación de los pensionistas en la fuerza laboral. Muchos beneficiarios optaron por dejar de trabajar, y se registró una mayor caída en la oferta laboral de aquellas personas autoempleadas para el caso de los varones y las formalmente empleadas en el caso de las mujeres. Asimismo, muchos de los beneficiarios que seguían trabajando continuaron haciéndolo, pero en el sector informal. De acuerdo con el estudio de Berniell (2014), la reforma tuvo un efecto positivo en el ingreso permanente de las mujeres y su contribución al ingreso total del hogar. Gracias a este incremento, los roles se hicieron más igualitarios dentro de sus hogares.

Por otro lado, Aramburú y Rodríguez (2011) mencionan que, entre los años 2004 y 2009, la pobreza a nivel nacional se redujo en un $28,2 \%$; sin embargo, para el mismo período, la desigualdad en la distribución del ingreso cambio mínimamente, de 0,49 a 0,48, según el coeficiente de Gini. La mejora de la distribución del ingreso supone políticas tributarias y de crecimiento "propobres» y mayores esfuerzos para mejorar el acceso de los hogares de menores ingresos a los bienes y servicios públicos. Disminuir la desigualdad es primordial para contribuir a una sociedad más cohesionada. Los programas focalizados de lucha contra la pobreza extrema deben concentrarse en las zonas rurales de sierra y selva.

La pobreza extrema presenta mayores diferencias regionales que la pobreza: es casi 140 veces mayor entre la población rural que en Lima; y 13 veces más alta en la sierra y 9 veces mayor en la selva, que en la costa. Sin embargo, las evaluaciones de impacto sobre el consumo con énfasis en el gasto per cápita de alimentos, son muy limitadas en nuestro país. De modo que el objetivo de esta investigación es estimar el impacto del programa social Pensión 65 sobre el gasto per cápita en alimentos. Con este fin, se utilizan la base de datos de la Encuesta Nacional de Hogares (Enaho) 
2017-2018, para identificar a los beneficiarios directos del programa (grupo de tratamiento) y construir un grupo de comparación con la metodología de doble diferencia con MCO y efectos fijos (EF) con datos de panel. La unidad de análisis son los hogares del Perú a nivel nacional que participan dos años consecutivos en dicho programa social, y se ha tomado como la línea de base el año 2017.

En este sentido, el presente artículo tiene como objetivo principal determinar el impacto del programa social Pensión 65 sobre el gasto per cápita en alimentos. La metodología utilizada se concentra en el análisis econométrico mediante la estimación del modelo de doble diferencia con propensity score matching (DD-PSM) con datos de panel de la Encuesta Nacional de Hogares (Enaho) 2017-2018. La estructura temática del trabajo está organizada de la siguiente manera: en la parte introductoria se discuten la problemática de la acción del programa Pensión 65 y los antecedentes teóricos y metodológicos desarrollados en los diferentes contextos referidos al tema de estudio. A continuación, se describe el proceso metodológico utilizado para el tratamiento de los datos y las estimaciones realizadas para determinar el impacto. La siguiente parte del trabajo está referida a las evidencias y discusiones de los resultados de impacto del programa Pensión 65 en el gasto per cápita en alimentos de los beneficiarios; $y$, finalmente, se presentan las conclusiones del estudio en relación con el objetivo planteado inicialmente.

\section{Métodos y materiales}

En la literatura sobre evaluación de impacto existen diversos métodos cuantitativos que abordan el problema de no aleatoriedad en los grupos de tratamiento y de control de un programa social. El uso de un método en particular obedece a la relevancia en un contexto en el cual el propósito de la política pública es incidir sobre un grupo focalizado de personas, como es el caso del programa Pensión 65, dirigido a adultos mayores de 65 años (Angrist \& Pischke, 2008). En lo que respecta a la estrategia metodológica, el estudio se propone utilizar dos variaciones del método de diferencias en diferencias clásico con la finalidad de definir grupos de control, para determinar la diferencia en el gasto per cápita en alimentos entre ambos grupos. Dada la naturaleza de los datos, en especial por el gasto en el consumo per cápita en alimentos por usuario, estas aproximaciones se estiman por mínimos cuadrados ordinarios con efectos fijos.

La principal fuente de información que se ha utilizado en la investigación es la Encuesta Nacional de Hogares (Enaho), que es elaborada todos los años por el Instituto Nacional de Estadística e Informática (INEI). Esta encuesta es representativa a nivel departamental y contiene información 
sobre horas trabajadas, estatus laboral, educación, pertenencia a programas sociales, materiales de vivienda, acceso a servicios básicos, entre otra, que es necesaria para el análisis de regresión presentado.

\section{Muestra}

La muestra incluye en el grupo de tratamiento a aquellos hogares que cuentan con un solo beneficiario y en los que ningún otro miembro participa en programas no alimentarios. En el grupo de control están aquellos hogares con miembros que no reciben ninguno de los programas sociales no alimentarios (véase la tabla 1). La muestra final incluye hogares de tipo panel de dos años (2017-2018).

Tabla 1

Requisitos para pertenecer al grupo de tratamiento o control

\begin{tabular}{|c|c|}
\hline Grupo de tratamiento & Grupo de control \\
\hline $\begin{array}{l}\text { A nivel de hogares, la persona solo debe } \\
\text { pertenecer al programa Pensión } 65 \text { y a ningún } \\
\text { otro programa social no alimentario. }\end{array}$ & $\begin{array}{l}\text { Todos los miembros del hogar no deben } \\
\text { pertenecer a ningún programa social no } \\
\text { alimentario. }\end{array}$ \\
\hline $\begin{array}{l}\text { El hogar debe contar solo con una persona } \\
\text { beneficiaria con } 65 \text { años a más. }\end{array}$ & $\begin{array}{l}\text { Se elige al jefe de hogar en pobreza o extrema } \\
\text { pobreza. }\end{array}$ \\
\hline
\end{tabular}

\section{Modelo econométrico para estimar el impacto con datos de panel}

En particular, la metodología de tipo cuasiexperimental propuesta por Ravallion (2007) y Rosenbaum y Rubin (1983) sugiere identificar el grupo de hogares que reciben las transferencias monetarias (grupo de tratamiento), denotado por PENSIÓN=1, y el grupo de hogares de similares características, pero que no reciben las transferencias (PENSIÓN=0); siguiendo a Zegarra (2015) y Shahidur et al. (2010), cuando el modelo econométrico es de tipo panel, para medir el impacto se usa la siguiente especificación para cada hogar en cada período $t$ :

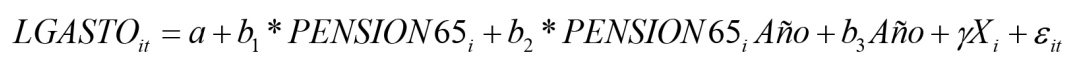

Dónde: LGASTO es el logaritmo del gasto per cápita en alimentos y $X_{i}$ es el vector de características observables que hacen diferente a cada hogar o adulto mayor. Asimismo, la especificación es aplicable a dos períodos: en el año 0, el hogar es la llamada «línea de base», mientras que en el año 1 está en el período de «medición de impactos». La variable PENSIÓN tiene el valor 1 si un hogar es tratado (tanto en la línea de base, cuando aún no recibe tratamiento, como en la medición posterior), y el valor 0 si un hogar no es tratado (también en línea de base y medición posterior). Los coeficientes $b_{1}, b_{2}$ y $b_{3}$ son cruciales para entender la dinámica de los efectos. El primer 
coeficiente, $b_{1}$, mide el valor medio de la diferencia entre hogares tratados y no tratados en la línea de base. Por otro lado, el coeficiente $b_{3}$ mide el cambio en la variable de impacto entre la línea de base y la medición posterior para todos los hogares. Finalmente, $b_{2}$ mide el impacto de DD del programa en la variable dependiente, es decir, es la medición de impacto del tratamiento en los tratados del programa, que es equivalente al impacto $\Delta \mathrm{Y}$. El modelo se puede estimar por efectos fijos (EF) con propensity score matching (PSM).

\section{Análisis de resultados}

En este acápite se presentan los resultados de las estimaciones del impacto del programa Pensión 65 en el gasto per cápita del consumo. En primer lugar, se discuten los resultados de las estimaciones según el modelo de doble diferencia con propensity score matching (DD-PSM) con efectos fijos por conglomerado. Asimismo, en este apartado se discute la consistencia de los resultados; por lo tanto, es poco probable que los cambios sean consecuencia de una heterogeneidad no observada entre los dos grupos. Los efectos del tratamiento promedio general son mayores en magnitud, pero no estadísticamente diferentes a las estimaciones de la intención de tratamiento en los participantes del programa. En resumen, comparamos los resultados con la inclusión de algunas covariables. Para los resultados individuales, las covariables incluyeron la condición de pobreza, edad, años de educación, tenencia de agua potable, necesidades básicas insatisfechas y zona de ubicación (urbana/rural) del individuo.

\section{Identificación del grupo de tratamiento y control}

En esta sección se describe el método de identificación de los grupos de tratamiento y de control para evaluar los efectos del programa en los usuarios. Por lo tanto, el principal reto empírico es crear un grupo que pueda modelar lo que habría sido de los usuarios sin el programa. Para ello, se explotó la variación exógena propuesta por el umbral de elegibilidad: un individuo es elegible solo si su puntuación está por debajo del umbral de elegibilidad (los umbrales varían a través de los 15 conglomerados en la muestra); estimamos el efecto del tratamiento promedio local; comparamos los resultados de aquellos con puntuaciones justo por debajo del umbral con los de aquellos justo por encima; y estimamos impactos introduciendo efectos fijos por conglomerado. Esto último pudiera parecer innecesario porque todos los umbrales caen dentro del rango de 0,3 de desviaciones estándar del puntaje Sisfoh. Sin embargo, los efectos fijos por conglomerados fueron pertinentes a nuestro modelo por dos razones. En primer lugar, modelaron la variación geográfica común del conglomerado. En segundo lugar, permitieron una estimación más eficiente. 
La base de datos de la Enaho reporta la lista de beneficiarios de los programas sociales no alimentarios; en esta base se puede identificar el número de beneficiarios por hogar. En la tabla 2 se muestra a aquellos hogares con 1,2 y hasta 3 miembros beneficiarios. Se puede constatar que el 20,35\% de los hogares de la muestra tienen dos (2) beneficiarios en el año 2017; sin embargo, para efectos de identificación del grupo de tratamiento no se tomó en cuenta a aquellos hogares con más de dos beneficiarios.

Tabla 2

Hogares con 1, 2, 3 y 4 miembros beneficiarios del programa Pensión 65

\begin{tabular}{ccccc}
\hline \multirow{2}{*}{ Beneficiarios } & \multicolumn{2}{c}{2017} & \multicolumn{2}{c}{2018} \\
\cline { 2 - 5 } & Hogares & $\%$ & Hogares & $\%$ \\
\hline 1 & 2.393 & 79,55 & 2.863 & 81,10 \\
2 & 612 & 20,35 & 665 & 18,84 \\
3 & 3 & 0,10 & 1 & 0,03 \\
4 & -- & & 1 & 0,03 \\
\hline Total & 3.008 & 100 & 3.530 & 100 \\
\hline
\end{tabular}

Fuente: elaboración propia con base en la Enaho 2017-2018.

Por otro lado, la base de datos de la Enaho reporta información sobre programas sociales a nivel de hogares. En esta base se puede identificar qué hogares son beneficiarios de los programas sociales no alimentarios. En el estudio para el grupo de tratamiento no se incluye a aquellos hogares que se benefician simultáneamente de varios programas sociales (Pensión 65, Juntos, Beca 18, etc.); concretamente, el grupo de tratamiento lo conforman únicamente aquellos hogares que reciben Pensión 65. En la tabla 3 se reporta para el año 2018 un total de 2.006 hogares beneficiarios de Pensión 65, que representan el 49,37\%. En el grupo de control están aquellos adultos cuyo hogar se encuentra en condición de pobreza y que cuentan con necesidades básicas insatisfechas (NBI).

Tabla 3

Grupo de tratamiento y control

\begin{tabular}{ccccc}
\hline \multirow{2}{*}{ Hogares } & \multicolumn{2}{c}{2017} & \multicolumn{2}{c}{2018} \\
\cline { 2 - 5 } & Número & $\%$ & Número & $\%$ \\
\hline Control & 1.893 & 54,81 & 2.057 & 50,63 \\
Tratamiento & 1.561 & 45,19 & 2.006 & 49,37 \\
\hline Total & 3.454 & 100 & 4.063 & 100 \\
\hline
\end{tabular}

Fuente: elaboración propia con base en la Enaho 2017-2018. 


\section{Estadística descriptiva del gasto en alimentos per cápita mensual: 2017-2018}

Según las especificaciones técnicas en el diseño del Programa Presupuestal, el resultado esperado del programa Pensión 65 es proporcionar seguridad económica a sus beneficiarios mayores de 65 años en situación pobreza extrema, que les permita mejorar su bienestar. Los indicadores de resultado específico definen tasa de actividad de los adultos mayores, número de horas de trabajo, incremento de su acceso a los servicios de salud y gasto promedio per cápita de los hogares. En la tabla 4 se evidencian los resultados de la comparación estadística mediante el logaritmo del gasto per cápita en alimentos mensuales (LGASTO) entre el grupo afiliados al programa (tratamiento) y no afiliados (control). Específicamente, para el año 2018, el LGASTO promedio en el grupo tratamiento fue de 4,168, mientras en el grupo de control fue de 4,507, una diferencia de 0,338 , que implica una diferencia de $33,8 \%$. Utilizando el estadístico de $t$, se rechaza la hipótesis nula $(\mathrm{Ho})$ de que los promedios son iguales a un nivel de significancia del 5\%, es decir, existen diferencias significativas en el gasto per cápita entre ambos grupos.

De manera similar, para el año 2017 existen diferencias en el gasto promedio per cápita (LGASTO) entre ambos grupos (tratamiento y control). Por otro lado, si se compara el gasto per cápita entre los años 2017 y 2018 entre el grupo de afiliados al programa, el efecto de Pensión 65 sobre el gasto promedio per cápita para los adultos mayores es positivo y significativo al $95 \%$ de confianza. De esta manera, ser beneficiario del programa incrementa su gasto promedio per cápita en un 1,9\% aproximadamente. Igualmente, si se compara el gasto promedio per cápita en ese mismo período en el grupo de control (no afiliados), el gasto per cápita se incrementó solo en un $0,4 \%$. Un dato importante es la doble diferencia entre los años 2017 y 2018: este resultado es $(-0,023)$. Se puede decir que, según el modelo de doble diferencia, el gasto per cápita en alimentos se ha reducido en un $2,3 \%$ en este período. 
Tabla 4

Diferencia en el promedio entre el grupo de hogares de tratamiento y el de control

\begin{tabular}{|c|c|c|c|c|}
\hline & & $\begin{array}{c}\text { Enaho } 2017 \\
{[1]}\end{array}$ & $\begin{array}{c}\text { Enaho } 2018 \\
{[2]}\end{array}$ & $\begin{array}{c}\text { Diferencia } \\
\text { [2]-[1] }\end{array}$ \\
\hline \multirow[t]{2}{*}{ (a) Afiliados a Pensión 65} & & 4,149 & 4,168 & 0,019 \\
\hline & $\mathrm{n}$ & 1.893 & 2.057 & \\
\hline \multirow[t]{2}{*}{ (b) No afiliados a Pensión 65} & & 4,511 & 4,507 & 0,004 \\
\hline & $\mathrm{n}$ & 1.561 & 2.006 & \\
\hline (a)-(b) & & $0,361^{* * *}$ & $0,338^{* * *}$ & $-0,023^{* * *}$ \\
\hline Error estándar & & 0,013 & 0,024 & 0,006 \\
\hline Estadístico $t$ & & 13,48 & 14,03 & 3,833 \\
\hline
\end{tabular}

${ }^{* * *} \mathrm{p}<0.01,{ }^{* *} \mathrm{p}<0.05,{ }^{*} \mathrm{p}<0.1$ (denotan significancia al nivel de $1 \%, 5 \%$, y $10 \%$, respectivamente). Fuente: elaboración propia con base en los datos de la Enaho 2017-2018.

\section{Ecuación de propensity score matching}

$\mathrm{Al}$ igual que en la anterior estimación con modelo de doble diferencia, el efecto de interés en esta especificación es el asociado a la interacción entre recibir Pensión 65 y ser pobre extremo. Por ello, la técnica de estimación de impacto de doble diferencia con propensity score matching (DD-PSM) implica, en primer lugar, identificar las variables o características observables del adulto, del hogar y de la vivienda que determinan la probabilidad de afiliarse al programa Pensión 65. Este propósito se logra estimando el modelo probit. En efecto, en la tabla 5 se reportan dos especificaciones del modelo probit. En la especificación [1] se incluyen todas las variables observables, y los signos de los coeficientes son los esperados; sin embargo, esta especificación no cumple con el supuesto de balanceo y soporte común, por lo que finalmente se logra estimar con la especificación [2], cuyos signos de los coeficientes son como se esperaba y consistentes estadísticamente; además, esta especificación permite lograr el cumplimiento del supuesto de soporte común en [0,0638, 0,9979], y el balanceo se logró con las variables observables en cinco bloques comunes, por lo que se garantiza la robustez de la estimación por DD-PSM. En efecto, de los resultados se puede inferir que aquellos adultos con mayor edad que residen en viviendas con necesidades básicas insatisfechas (NBI) y que pertenecen a hogares pobres, tienen mayor probabilidad de acceder al programa Pensión 65. Asimismo, aquellos adultos mayores que han alcanzado menos años de educación y pertenecen a hogares en situación de pobreza, tienen mayor probabilidad de afiliarse al programa (figura 1). Por otro lado, aquellos adultos mayores que no tienen acceso a agua potable y residen en la zona rural, tienen mayor 
probabilidad de ser considerados como población objetivo del programa Pensión 65.

Figura 1

Efecto marginal de la pobreza sobre la probabilidad de participar en el programa Pensión 65

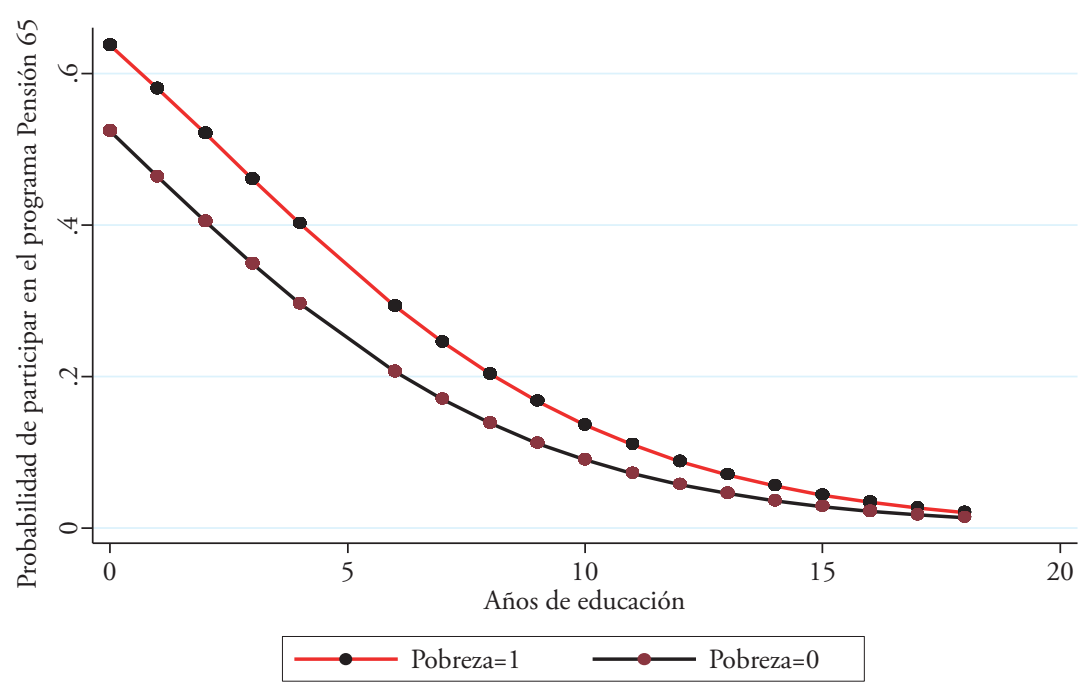

Tabla 5

Determinantes de la probabilidad de afiliarse al programa Pensión 65

\begin{tabular}{lcc}
\hline \multirow{2}{*}{ Variables } & \multicolumn{2}{c}{ Ecuaciones de propensity score } \\
\cline { 2 - 3 } & {$[1]$} & {$[2]$} \\
\hline Pobreza=1 & $0,26783^{* *}$ & $0,259711^{* *}$ \\
Años de educación & $(0,10987)$ & $(0,10900)$ \\
& $-0,110114^{* * *}$ & $-0,113003^{* * *}$ \\
Edad en años & $(0,01414)$ & $(0,01409)$ \\
& $0,02242^{* * *}$ & $0,023208^{* * *}$ \\
nbi1 & $(0,00714)$ & $(0,00711)$ \\
& $0,743225^{* * *}$ & $0,780554^{* * *}$ \\
nbi2 & $(0,218168)$ & $(0,21645)$ \\
& $1,42011^{* * *}$ & $1,417726^{* * *}$ \\
nbi3 & $(0,48358)$ & $(0,46895)$ \\
& $0,73868^{* * *}$ & \\
nbi4 & $(0,19919)$ & \\
& 0,281641 & \\
& $(0,85141)$ &
\end{tabular}




\begin{tabular}{lcc} 
nbi5 & $-0,242423$ & \\
\hline Agua potable $=1$ & $(0,81660)$ & \\
& $-0,65604^{* * *}$ & $-0,703476^{* * *}$ \\
Urbano=1 & $(0,12080)$ & $(0,11877)$ \\
& $-0,7451144^{* * *}$ & $-0,798241^{* * *}$ \\
_cons & $(0,13198)$ & $(0,12961)$ \\
& $-0,812664$ & $-0,739228$ \\
\hline Observaciones & $(0,548983)$ & $(0,54548)$ \\
Razón de verosimilitud & 1.068 & 1.068 \\
Pseudo $\mathrm{R}^{2}$ & 369,42 & 354,97 \\
\hline
\end{tabular}

${ }^{* * *} \mathrm{p}<0.01,{ }^{* *} \mathrm{p}<0.05,{ }^{*} \mathrm{p}<0.1$ (denotan significancia al nivel de $1 \%, 5 \%$, y $10 \%$, respectivamente). Fuente: elaboración propia con base en los datos de la Enaho 2017-2018.

\section{- Descripción de los datos de panel de la Enaho}

A fin de medir los cambios en el comportamiento de algunas características de la población, se ha implementado una muestra tipo panel. Estos cambios se refieren a datos que combinan series de tiempo con corte transversal. La dimensión temporal enriquece la estructura de los datos y es capaz de aportar información que no aparece en un único corte. Específicamente, el Instituto Nacional de Estadística e Informática (INEI), dentro de las encuestas anuales que aplica la Enaho, administra una submuestra panel; esto quiere decir que un grupo de hogares es entrevistado al ańo siguiente y se convierte en muestra de hogares panel con dos observaciones anuales. Cada año se vuelve a seleccionar aleatoriamente una submuestra, independientemente de la anterior. Esta metodología permite generar paneles de dos, tres o más años. En la tabla 6 se muestra el resumen de los datos balanceados de tipo panel con dos años. En cierta medida, el tamaño de la muestra es pequeño, pero la estimación del impacto podría ser consistente en comparación con la línea de base (año 2017)

Tabla 6

Datos de paneles con dos ańos

\begin{tabular}{ccc}
\hline \multirow{2}{*}{ Hogares } & \multicolumn{2}{c}{$2017-2018$} \\
\cline { 2 - 3 } & Numero de observaciones & $\%$ \\
\hline Control & 420 & 40,94 \\
Tratamiento & 606 & 59,06 \\
\hline Total & 1.026 & 100 \\
\hline
\end{tabular}

Fuente: elaboración propia con base en los datos de la Enaho 2017-2018. 


\section{- Impacto del programa Pensión 65 sobre el gasto per cápita con panel 2017-2018}

En la tabla 7 se muestra la estimación del impacto del programa Pensión 65 mediante la técnica de doble diferencia (DD) tomando como línea de base el año 2017. El resultado es de 0,1204 y es estadísticamente significativo a un nivel de significancia del 10\%; esto implica que el gasto per cápita en alimentos para el grupo de afiliados al programa aumentó en un 12,04\%. Sin embargo, esta forma de estimar el impacto solo es válida siempre y cuando la asignación del programa sea aleatoria. En la práctica, la asignación del programa no es aleatoria: más bien es condicionada a un conjunto de características observables del hogar y del adulto mayor. Así, el programa focaliza a quiénes atender mediante el Sisfoh y toma en cuenta un conjunto de variables, entre las cuales se encuentran requisitos como: estar en situación de pobreza, tener necesidades básicas insatisfechas, tener 65 años o más, etc.

Tabla 7

Doble diferencia con línea de base del año 2017

\begin{tabular}{lc}
\hline & LGASTO \\
\hline (a) Afiliados a Pensión 65 & 0,01268 \\
$\mathrm{n}$ & 308 \\
(b) No afiliados a Pensión 65 & $-0,1077$ \\
$\mathrm{n}$ & 205 \\
(a)-(b) & $0,1204^{*}$ \\
Error estándar & 0,0695 \\
Estadístico t & 1,82 \\
\hline
\end{tabular}

${ }^{* * *} \mathrm{p}<0.01,{ }^{* *} \mathrm{p}<0.05,{ }^{*} \mathrm{p}<0.1$ (denotan significancia al nivel de $1 \%, 5 \%$, y $10 \%$, respectivamente). Fuente: elaboración propia con base en los datos de la Enaho 2017-2018.

En la tabla 8 y la figura 1 se reporta la estimación del impacto del programa Pensión 65 a través de la estimación de regresiones aplicando el método de efectos fijos ${ }^{1}$ de datos de panel con dos períodos (2017-2018), tomando como la línea de base el año 2017. Por un lado, la técnica de DD reporta el mismo resultado que fue obtenido en la tabla 8 , es decir, el coeficiente de 0,1204 de la regresión mide el impacto del programa suponiendo que la asignación del beneficio es aleatoria. Sin embargo, la técnica de estimación también de efectos fijos de DD con PSM con errores estándar clusterizados

1 Estos modelos sirven para controlar la heterogeneidad inobservable, en particular cuando esta es constante en el tiempo y está correlacionada con las variables independientes. El supuesto del modelo de efectos fijos es que el efecto específico individual está correlacionado con las variables independientes. 
por hogares resulta más robusta y consistente porque toma en cuenta las características observables en el momento de asignar el beneficio mediante la ecuación de propensity score (modelo probit), la cual permite reducir el sesgo de selección y así comparar el resultado final bajo un soporte común en $[0,0638,0,9979]$, y el balanceo se logró con las variables observables en cinco bloques comunes. Asimismo, se logró controlar la heterogeneidad entre hogares incluyendo variables explicativas (pobreza, años de educación, edad, NBI, agua potable). Finalmente, el impacto de Pensión 65 sobre el gasto per cápita en alimentos es del 15\% aproximadamente, este coeficiente de impacto $\left(b_{2}\right)$ es positivo y significativo a un nivel de significancia del $10 \%$; por consiguiente, para el grupo de afiliados al programa, el gasto per cápita en alimentos del hogar se ha incrementado en un $15 \%$.

Tabla 8

Impacto del Programa Pensión 65 con el panel 2017-2018

\begin{tabular}{lcc}
\hline LGASTO & DD & DD-PSM \\
\hline Año & $-0,1077^{* *}$ & $-0,1148^{* *}$ \\
& $(0,0513)$ & $(0,0556)$ \\
PENSION65*Año & $0,1204^{*}$ & $0,1502^{*}$ \\
Pobreza=1 & $(0,0662)$ & $(0,09079)$ \\
& & $-0,6749$ \\
Años de educación & & $(0,1228)$ \\
& & 0,00163 \\
Edad en años & & $(0,01672)$ \\
& & 0,0062 \\
NBI1 & & $(0,0075)$ \\
& & $-0,0558$ \\
NBI2 & & $(0,1436)$ \\
& & 0,1020 \\
Agua potable=1 & & $(0,1644)$ \\
Constante & & 0,1169 \\
Observaciones & $2,26^{*}$ & $(0,0914)$ \\
F & & $4,2977^{* * *}$ \\
\hline
\end{tabular}

Errores estándar clusterizados por hogar entre paréntesis.

${ }^{* * *} \mathrm{p}<0.01,{ }^{* *} \mathrm{p}<0.05,{ }^{*} \mathrm{p}<0.1$ (denotan significancia al nivel de $1 \%, 5 \%$, y $10 \%$, respectivamente).

Fuente: Elaboración propia con base a los datos ENAHO-2017-201 
En esencia, la estimación de doble diferencia usa tanto el cambio en el tiempo del grupo no tratado como una estimación del contrafactual para el cambio en el tiempo del grupo tratado. El supuesto clave es que, sin el programa, la tendencia en los dos grupos hubiera sido igual. Esto es el supuesto de tendencias comunes o de tendencias paralelas. En el caso del programa Pensión 65 para personas adultas de 65 ańos a más, el supuesto implicaría que, sin la ayuda adicional, las personas con programa y sin programa hubieran mejorado su calidad de vida al mismo ritmo. Pero es posible que, aun sin el programa, las personas hubieran mejorado. En la figura 2 se muestran la estimación contrafactual y el impacto logrado por el programa en la variable de estudio.

Figura 2

Impacto del programa Pensión 65 con linea de base 2017

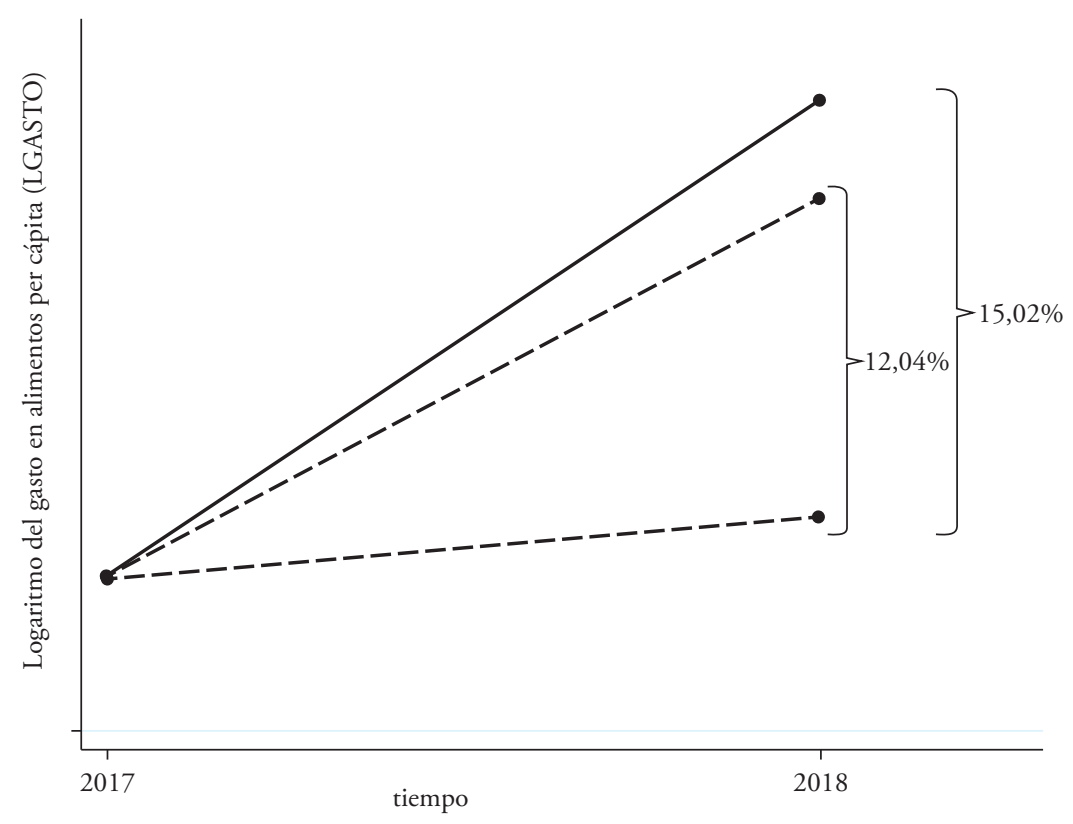

\section{Conclusiones}

Los resultados en este estudio reflejan que la pensión no contributiva (Pensión 65) implementada por el Estado peruano evidencia mejoras en algunos indicadores del bienestar de los hogares beneficiarios, principalmente en el gasto en consumo. Asimismo, es lógico deducir que no solo se beneficiaron del programa aquellos que estuvieron afiliados (personas de 65 años a más), sino también a otros miembros del hogar a través del consumo. 
Los resultados del modelo de doble diferencia con propensity score matching (DD-PSM) evidencian un impacto positivo del programa en el incremento sobre el gasto per cápita en alimentos en los hogares beneficiarios. Los beneficiarios del programa Pensión 65 han logrado incrementar su gasto per cápita en alimentos en un 15,02\%, y el resultado es estadísticamente significativo a un nivel del $10 \%$. Este resultado supone que los afiliados al programa no están destinando la mayor parte del subsidio prioritariamente al gasto en alimentos. En conclusión, el programa Pensión 65 es una política pública efectiva para mejorar el bienestar social en la vejez y, principalmente, a través de un incremento en el consumo en el hogar, lo cual contribuye a reducir la pobreza extrema en el país.

El análisis del impacto del programa Pensión 65 no finaliza con el presente estudio, teniendo en cuenta que el contexto socioeconómico del país es diferencialmente complejo. Como propuesta de investigaciones futuras, se sugiere la aplicación de nuevas metodologías capaces de combinar datos cuantitativos y cualitativos para identificar la influencia en la decisión de gastos que realizan las personas beneficiarias de los programas de transferencias monetarias tanto solidarias como condicionadas; también se propone estudiar la presencia de factores que se relacionan con el bienestar y la mejora de la calidad de vida de los beneficiarios del programa a nivel nacional. 
Impacto del programa social Pensión 65 sobre el gasto en alimentos, 2017-2018

\section{Referencias}

Angrist, J. (1998). Estimating the labor market impact of voluntary military service using social security data on military applicants. Econometrica, 66(2), 249-288.

Angrist, J., \& Pischke, J. (2008). Mostly harmless econometrics: An empiricistís companion. Recuperado de http://58.27.242.36:8000/jspui/bitstream/1/146/1/Mostly\%20 harmless\%20econometrics.pdf

Aramburú, C., \& Rodríguez, M. N. (2011). Politicas sociales y pobreza. Lima, Perú.

Barrientos, A. (2003). What is the impact of non-contributory pensions on poverty? Estimates from Brazil and South Africa. Reino Unido: Institute for Development Policy and Management, University of Manchester.

Barrientos, A., \& Lloyd-Sherlock, P. (2002). Non-contributory pensions and social protection. Paper for the series «Issues in Social Protection», published by the Social Protection Sector, International Labour Organization.

Berniell, I. (2014). The impact of a permanent income shock on the situation of women in the household: The case of a pension reform in Argentina. Argentina: Cemfi.

Bosch, M., \& Guajardo, J. (2012). Labor market impacts of non-contributory pensions: The case of Argentina's Moratorium. Inter-American Development Bank, Labor Markets and Social Security Unit.

Carvalho Filho, I. E. (2012). Household income as a determinant of child labor and school enrollment in Brazil: Evidence from a social security reform. Economic Development and Cultural Change, 60(2), 399-445.

Dirección de Calidad del Gasto Público del Ministerio de Economía y Finanzas. (2015). Nota metodológica para la evaluación de impacto del Programa Nacional de Asistencia Solidaria "Pensión 65" (MEF y Midis, 2013).

Durán Valverde, F., Mendoza de Souza, W., \& Picado Chacón, G. (2009). Proyecciones demográficas y financieras para el análisis de la viabilidad de las pensiones no contributivas en el Perú. En UNFPA, OIT, Cáritas del Perú, \& HelpAge International (Eds.), Envejecimiento con dignidad: pensiones no contributivas para reducir la pobreza en el Perú (pp. 37-87). Lima.

Edmonds, E. (2006). Child labor and schooling responses to anticipated income in South Africa. Journal of Development Economics, 81(2), 386-414.

Galiani, S., \& Gertler, P. (2012). Primer seguimiento a la evaluación de impacto del Programa de Atención a Adultos Mayores de 70 años y más en zonas rurales (Programa 70 y Más). Sedesol.

Galiani, S., Gertler, P., \& Bando, R. (2014). Non-contributory pensions. IDB Working Paper Series 517. Washington D. C.: Inter-American Development Bank.

Gondim Teixeira, C. (2009). ¿Cuál es el impacto de las transferencias monetarias sobre la oferta de mano de obra? Brasil: International Policy Centre for Inclusive Growth.

Leveau, E., \& Marín, J. (2015). Impacto del programa Pensión 65 en el bienestar del adulto mayor de la ciudad de Tarapoto, periodo 2012-2014. Perú.

Olivera, J., \& Clausen, J. (2014). Las características del adulto mayor peruano y las políticas de protección social / Features of the Peruvian elderly and social protection policies. Economia, 37(73), 75.

Ravallion, M. (2007). Evaluating anti-poverty programs. Handbook of Development Economics, 4, 3787-3846. 
Rosenbaum, R., \& Rubin, D. (1983). The central role of the propensity score in observational studies for causal effects. Biometrika, 70(1), 41-55.

Shahidur, R. et al. (2010). Handbook on impact evaluation. Quantitative methods and practices. The World Bank.

Teixeira, C. G. (2008). Análise do impacto do Programa Bolsa Família na oferta de trabalho dos homens e mulheres. Recuperado de https://ipcig.org/publication/mds/27P.pdf

Torres, J., \& Salinas, C. (2016). Impacto laboral potencial del acceso a Pensión 65: un primer análisis. Lima: CIES.

Zegarra, E. (2015). Efectos dinámicos del programa Juntos en decisiones productivas de los hogares rurales del Perú. Lima: Grade. 